\title{
Quantum Stochastic Processes III
}

\author{
E. B. Davies * \\ Mathematical Institute, Oxford \\ Received October 26, 1970
}

\begin{abstract}
We construct an example of a quantum stochastic process with a non-zero, linear, time-independent source, for a massive scalar Boson field in four space-time dimensions. Also we study in detail a similar process with only a single degree of freedom.
\end{abstract}

\section{§ 1. Introduction and Summary of Results}

In an earlier paper [1] we laid the foundations for a theory of quantum stochastic processes, in order to provide a mathematical framework for the description of the evolution of a free photon field in the presence of localised external sources and localised absorptive photon detectors. At the end of that paper a model was constructed which was adequate for arbitrary Boson fields provided sources were absent.

In this paper we construct an example with a non-zero, linear, timeindependent source. This is done for a massive scalar Boson field in four space-time dimensions, but formally we are describing the same situation as has been considered non-rigorously in quantum optics. The theory has two space cut-offs, corresponding to the finite extents of the particle source (or sources) and the particle detector (or detectors). As is conventional in quantum optics we suppose that the particle detector is purely absorptive, so the theory is not strictly local.

The same construction allows us to consider the behaviour of a displaced harmonic oscillator with external damping. By studying the time evolution of the coherent states we prove that all initial states converge to a unique equilibrium state, which is a pure coherent state and a slight perturbation of the natural ground state of the oscillator.

As far as the technical details are concerned we point out that for time-independent linear sources the complications can be reduced by making a canonical transformation of the Hilbert space. However, since a realistic model must incorporate time-dependent sources, that method would lead to the necessity of studying randomly fluctuating canonical transformations. With the method we adopt, however, the inclusion of fluctuations in the source is a relatively straightforward matter.

* Work supported by A.F.O.S.R. contract no. F44620-67-C-0029 
We denote by $X$ the locally compact abelian group $\mathbb{R}^{3}$ with Lebesgue measure $d x$, taken as position space, and by $K$ the same group taken as momentum space. We define Fock space $\mathscr{F}=\sum_{n=0}^{\infty} \oplus \mathscr{F}^{(n)}$ where $\mathscr{F}^{(n)}=L_{\text {sym }}^{2}\left(K^{n}\right)$. We define $\omega(k)=\left(m_{0}^{2}+k_{1}^{2}+k_{2}^{2}+k_{3}^{2}\right)^{1 / 2}$ on $K$ so that

$$
H_{0}=\int \omega(k) a^{*}(k) a(k) d k
$$

is the free Hamiltonian. The total Hamiltonian of the system due to an external scalar current localized in various sources is

$$
H=H_{0}+H_{1}
$$

where

$$
H_{1}=-\frac{1}{\sqrt{2}} \int \omega(k)^{-1 / 2}\left[\overline{j(k)} a(k)+j(k) a^{*}(k)\right] d k
$$

and $j$ is supposed to be a function in Schwarz space.

In [1] we have shown how to construct a quantum stochastic process $\mathscr{E}$ from a Hamiltonian $H$ and a bounded stochastic kernel $\mathscr{J}$, which describes the interaction between the quantum field and the particle detector. We take $\mu$ to be a non-negative, real $C^{\infty}$-function of compact support on $X$ which is constant on the regions where the detectors are localized and equal to zero just outside. The annihilation operator at a point $x \in X$ is defined by

$$
A(x)=\frac{1}{\sqrt{2}}(2 \pi)^{-3 / 2} \int e^{-i x \cdot k} \omega(k)^{-1 / 2} a(k) d k .
$$

The stochastic kernel $\mathscr{J}$ is now defined formally for all Borel sets $E \cong X$ and all $\varrho \in \mathscr{T}_{s}(\mathscr{F})$ by

$$
\mathscr{J}(E, \varrho)=\int_{x \in E} \mu(x) A(x) \varrho A^{*}(x) d x .
$$

If $\varrho=\psi \otimes \bar{\psi}$ is a pure state, this may be rewritten as

$$
\begin{aligned}
& \mathscr{J}(E, \psi \otimes \bar{\psi}) \\
& \quad=\frac{1}{2}(2 \pi)^{-3} \iiint \frac{\mu(x) e^{-i x \cdot(k-h)}}{\omega(k)^{1 / 2} \omega(h)^{1 / 2}} \chi_{E}(x)\{a(k) \psi\} \\
& \otimes\{a(h) \psi\}^{-} d k d h d x .
\end{aligned}
$$

The interaction rate $R$ of $\mathscr{J}$ is the operator on $\mathscr{F}$ such that

$$
\operatorname{tr}[\mathscr{J}(X, \varrho)]=\operatorname{tr}[R \varrho]
$$


for all $\varrho \in \mathscr{T}_{s}(\mathscr{F})$, and formally

$$
\begin{aligned}
R & =\int_{x} \varrho(x) A^{*}(x) A(x) d x \\
& =\frac{1}{2}(2 \pi)^{-3 / 2} \iint \frac{\tilde{\mu}(k-h)}{\omega(k)^{1 / 2} \omega(h)^{1 / 2}} a^{*}(h) a(k) d k d h
\end{aligned}
$$

where $\tilde{\mu}$ is the Fourier transform of $\mu$. $R$ is an unbounded positive operator which commutes with the number operator $N$. Formally, as in [1], we expect that

$$
\mathscr{E}_{t}(z, \varrho)=B_{t} \varrho B_{t}^{*}
$$

where $z \in X_{t}$ is the point corresponding to no events having occurred up to time $t$ and $B_{t}$ is the strongly continuous contraction semigroup on $\mathscr{F}$ whose infinitesimal generator is

$$
Z=-i H-\frac{1}{2} R \text {. }
$$

We construct this semigroup rigorously and obtain some estimates of its behaviour in time.

In order to proceed we introduce a particle number cut-off into the interaction. For any $\delta>0$ we show that

$$
\mathscr{J}^{\delta}(E, \varrho)=\mathscr{J}\left(E, e^{-\delta N} \varrho e^{-\delta N}\right)
$$

is well-defined as a bounded stochastic kernel on $X$ whose interaction rate is the operator

$$
R^{\delta}=e^{-2 \delta N} R \leqq R .
$$

By the construction of [1] we obtain from $Z$ and $\mathscr{J}^{\delta}$ a substochastic process $\mathscr{E}_{t}^{\delta}$, by which we mean the same as a quantum stochastic process except that the condition

$$
\operatorname{tr}\left[\mathscr{E}_{t}\left(X_{t}, \varrho\right)\right]=\operatorname{tr}[\varrho]
$$

of [1] is replaced by

$$
\operatorname{tr}\left[\mathscr{E}_{t}^{\delta}\left(X_{t}, \varrho\right)\right] \leqq \operatorname{tr}[\varrho]
$$

valid for all $\varrho \in \mathscr{T}_{s}(\mathscr{F})^{+}$and all $t \geqq 0$. By making various estimates on the behaviour of $\mathscr{E}_{t}^{\delta}$ we show that there exists a quantum stochastic process $\mathscr{E}_{t}$ on $X, \mathscr{T}_{s}(\mathscr{F})$ such that for all $\varrho \in \mathscr{T}_{s}(\mathscr{F})$ and all $E \leqq X_{t}$

$$
\mathscr{E}_{t}(E, \varrho)=\lim _{\delta \rightarrow 0} \mathscr{E}_{t}^{\delta}(E, \varrho)
$$

provided $t$ is sufficiently small. 
The estimates are obtained by introducing a doubly indexed family of norms in $\mathscr{F}$. We first choose $\gamma_{0}>0$ large enough so that

$$
\int \omega(k)^{-\gamma_{0}} d k<\infty \text {. }
$$

Then for each $\gamma \geqq \gamma_{0}$ we define $D_{\gamma}^{(n)}$ as the space of functions in $\mathscr{F}^{(n)}$ such that the norm

$$
\|\psi\|_{\gamma}^{(n)}=\sup \left\{\left|\psi\left(k_{1} \ldots k_{n}\right)\right| m_{0}^{-n \gamma} \omega\left(k_{1}\right)^{\gamma} \ldots \omega\left(k_{n}\right)^{\gamma}\right\}
$$

is finite. For each $\alpha>0$ we define $D_{\gamma, \alpha}$ as the space of sequences $\left\{\psi^{(n)} \in D_{\gamma}^{(n)}\right\}_{n=0}^{\infty}$ such that the norm

$$
\|\psi\|_{\gamma, \alpha}=\sup _{n}\left\{\|\psi\|_{\gamma}^{(n)} e^{\alpha n}\right\}
$$

is finite. There exist numbers $c_{0}$ and $\alpha_{0}$ such that $D_{\gamma_{0}, \alpha_{0}}$ is contained in $\mathscr{F}$ and

$$
\|\psi\| \leqq c_{0}\|\psi\|_{\gamma_{0}, \alpha_{0}}
$$

for all $\psi \in D_{\gamma_{0}, \alpha_{0}}$. If $\gamma \leqq \gamma^{\prime}$ and $\alpha \leqq \alpha^{\prime}$ then

$$
\|\psi\|_{\gamma, \alpha} \leqq\|\psi\|_{\gamma^{\prime}, \alpha^{\prime}}
$$

for all $\psi \in D_{\gamma^{\prime}, \alpha^{\prime}}$. We define $D_{\gamma, \alpha}=\bigcap_{\alpha} D_{\gamma, \alpha}$ and $\mathscr{D}=\bigcap_{\gamma} D_{\gamma, \infty}$, both being topologized by using all the available (finite) norms simultaneously. All the constants in the next section will depend on $m_{0}, j, \mu, \gamma_{0}, \alpha_{0}, c_{0}$, and $\gamma$, but we shall not keep referring to this fact.

\section{$\S 2$. Construction of the Semigroup $B_{t}$}

In order to obtain the growth estimates we need for $B_{t}$, we have to construct it in two stages. First note that

$$
\begin{gathered}
\left(e^{-i H_{0} t} \psi\right)^{(n)}\left(k_{1} \ldots k_{n}\right) \\
=e^{-i \omega\left(k_{1}\right) t} \ldots e^{-i \omega\left(k_{n}\right) t} \psi^{(n)}\left(k_{1} \ldots k_{n}\right)
\end{gathered}
$$

so $e^{-i H_{0} t}$ is a one parameter group of isometries on each of the Banach spaces $D_{\gamma, \alpha}$. The operator $R$ of Eq. (1.9) is given explicitly by

$$
\begin{gathered}
(R \psi)^{(n)}\left(k_{1} \ldots k_{n}\right) \\
=n S_{n} \frac{1}{2}(2 \pi)^{-3 / 2} \int \frac{\tilde{\mu}\left(k-k_{1}\right)}{\omega\left(k_{1}\right)^{1 / 2} \omega(k)^{1 / 2}} \psi^{(n)}\left(k_{1} k_{2} \ldots k_{n}\right) d k
\end{gathered}
$$

where $S_{n}$ is the symmetrization projection on $L^{2}\left(K^{n}\right)$. By means of the estimates

$$
|\tilde{\mu}(k)| \leqq c_{1} \omega(k)^{-\gamma}
$$


and

$$
\int \omega(k-h)^{-\gamma} \omega(h)^{-\gamma} d h \leqq c_{2} \omega(k)^{-\gamma}
$$

valid for all $\gamma \geqq \gamma_{0}$, we obtain

so that

$$
\|R \psi\|_{\gamma}^{(n)} \leqq 2 c n\|\psi\|_{\gamma}^{(n)}
$$

$$
\begin{aligned}
\|R \psi\|_{\gamma, \alpha} & \leqq 2 c\|\psi\|_{\gamma, \alpha+1} \sup _{n}\left\{n e^{-n}\right\} \\
& \leqq 2 c\|\psi\|_{\gamma, \alpha+1} .
\end{aligned}
$$

We define the semigroup $C_{t}=e^{\left(-i H_{0}-\frac{1}{2} R\right) t}$ for all $t \geqq 0$ by its formal perturbation series

$$
C_{t}=e^{-i H_{0} t}+\sum_{m=1}^{\infty} C_{t}^{(m)}
$$

where

$$
\begin{gathered}
C_{t}^{(m)}=\left(-\frac{1}{2}\right)^{m} \int_{t_{1}=0}^{t} \ldots \int_{t_{m}=0}^{t_{m-1}} e^{-i H_{0}\left(t-t_{1}\right)} R \ldots \\
R e^{-i H_{0} t_{m}} d t_{1} \ldots d t_{m} .
\end{gathered}
$$

Lemma 2.1. The above formulae define $C_{t}$ as a semigroup on each space $D_{\gamma}^{(n)}$ for $\gamma \geqq \gamma_{0}$ and as a semigroup commuting with the number operator on $D_{\gamma, \alpha}$.

Proof. From Eqs. (2.5) and (2.8) we obtain the estimate

$$
\left\|C_{t}^{(m)} \psi\right\|_{\gamma}^{(n)} \leqq\left(\frac{1}{2}\right)^{m}(2 c n)^{m} \frac{t^{m}}{m !}\|\psi\|_{\gamma}^{(n)}
$$

so the series (2.7) converges in norm within $D_{\gamma}^{(n)}$ and

$$
\left\|C_{t} \psi\right\|_{\gamma}^{(n)} \leqq e^{c n t}\|\psi\|_{\gamma}^{(n)}
$$

from which one obtains

$$
\left\|C_{t} \psi\right\|_{\gamma, \alpha} \leqq\|\psi\|_{\gamma, \alpha+c t}
$$

which proves that $C_{t}$ leaves $D_{\gamma, \infty}$ invariant. That $C_{t}$ is a semigroup on $D_{\gamma}^{(n)}$ follows from the equation

$$
C_{s+t}^{(m)}=\sum_{p+q=m} C_{s}^{(p)} C_{t}^{(q)}
$$

which may be verified directly from Eq. (2.8) as in [5]. 
The operator $H_{1}: \mathscr{F}^{(n)} \rightarrow \mathscr{F}^{(n-1)} \oplus \mathscr{F}^{(n+1)}$ of Eq. (1.3) is explicitly given by

$$
\begin{gathered}
\left(H_{1} \psi\right)^{(n-1)}\left(k_{1} \ldots k_{n-1}\right) \\
=-\sqrt{\frac{n}{2}} \int \frac{\overline{j(k)}}{\omega(k)^{1 / 2}} \psi^{(n)}\left(k, k_{1} \ldots k_{n-1}\right) d k, \\
\left(H_{1} \psi\right)^{(n+1)}\left(k_{1} \ldots k_{n+1}\right) \\
=-\frac{1}{\sqrt{2(n+1)}} \sum_{r=1}^{n+1} \frac{j\left(k_{r}\right)}{\omega\left(k_{r}\right)^{1 / 2}} \psi^{(n)}\left(k_{1} \ldots \hat{k}_{r} \ldots k_{n+1}\right)
\end{gathered}
$$

where $\hat{k}_{r}$ indicates that this variable is omitted. Since $j$ is supposed to lie in Schwarz space we have estimates

$$
\left\|H_{1} \psi\right\|_{\gamma}^{(n-1)} \leqq c_{3} \sqrt{n}\|\psi\|_{\gamma}^{(n)}
$$

and

$$
\left\|H_{1} \psi\right\|_{\gamma}^{(n+1)} \leqq c_{3} \sqrt{n+1}\|\psi\|_{\gamma}^{(n)}
$$

valid for all $\gamma \geqq \gamma_{0}$, from which one obtains

$$
\begin{aligned}
& \left\|H_{1} \psi\right\|_{\gamma, \alpha} \\
& \leqq c_{3}\|\psi\|_{\gamma, \alpha+1} \sup _{n} e^{\alpha n}\left\{\sqrt{n+1} e^{-(\alpha+1)(n+1)}+\sqrt{n} e^{-(\alpha+1)(n-1)}\right\} \\
& \leqq 2 c_{3} e^{\alpha}\|\psi\|_{\gamma, \alpha+1} .
\end{aligned}
$$

We define the semigroup $B_{t}=e^{Z t}$ by its formal perturbation series

where

$$
B_{t}=C_{t}+\sum_{m=1}^{\infty} B_{t}^{(m)}
$$

$$
B_{t}^{(m)}=(-i)^{m} \int_{t_{1}=0}^{t} \ldots \int_{t_{m}=0}^{t_{m-1}} C_{t-t_{1}} H_{1} \ldots H_{1} C_{t_{m}} d t_{1} \ldots d t_{m} .
$$

To prove this sum converges we follow the estimates of Lanford [5], noting that

$$
B_{t}^{(m)}: \mathscr{F}^{(n)} \rightarrow \mathscr{F}^{(n-m)} \oplus \cdots \oplus \mathscr{F}^{(n+m)} .
$$

Lemma 2.2. The above formulae define $B_{t}$ as a semigroup on the space $D_{\gamma, \infty}$ provided $\gamma \geqq \gamma_{0}$.

Proof. If $\psi \in \mathscr{F}^{(r)}$ then

$$
\begin{aligned}
\left\|B_{t}^{(m)} \psi\right\|_{\gamma}^{(n)} \leqq & \int_{t_{1}=0}^{t} \ldots \int_{t_{m}=0}^{t_{m-1}} e^{c(r+m)\left(t-t_{1}\right)} c_{3} \sqrt{r+m} \ldots \\
& \ldots c_{3} \sqrt{r+1} e^{c r t_{m}}\|\psi\|_{\gamma}^{(r)} d t_{1} \ldots d t_{m} \\
\leqq & e^{c(r+m) t} c_{3}^{m}(r+m)^{m / 2} \frac{t^{m}}{m !}\|\psi\|_{\gamma}^{(r)}
\end{aligned}
$$


Now let $\beta=\alpha+c t+s$ and let $\psi \in D_{\gamma, \beta}$ where $\gamma \geqq \gamma_{0}$. Then

$$
\begin{aligned}
e^{\alpha n} \| B_{t}^{(m)} & \psi \|_{\gamma}^{(n)} \\
& \leqq \sum_{r=n-m}^{n+m} e^{\alpha n}\left\|B_{t}^{(m)} \psi^{(r)}\right\|_{\gamma}^{(n)} \\
& \leqq e^{\alpha n} e^{c(n+2 m) t} c_{3}^{m}(n+2 m)^{m / 2} \frac{t^{m}}{m !} \sum_{r=n-m}^{n+m}\|\psi\|_{\gamma}^{(r)} \\
& \leqq e^{\alpha n} e^{c(n+2 m) t} c_{3}^{m}(n+2 m)^{m / 2} \frac{t^{m}}{m !}(2 m+1) e^{-\beta(n-m)}\|\psi\|_{\gamma, \beta} \\
& \leqq(2 m+1) \frac{t^{m}}{m !} c_{3}^{m} e^{(2 c t+\beta) m}\|\psi\|_{\gamma, \beta} \sup _{n}\left\{e^{-s n}(n+2 m)^{m / 2}\right\} .
\end{aligned}
$$

Therefore

$$
\left\|B_{t}^{(m)} \psi\right\|_{\gamma, \alpha} \leqq \frac{(2 m+1) m^{m / 2} t^{m}}{m !} c_{3}^{m} e^{(2 c t+\beta) m}\left\{\max \left(2, \frac{1}{2 s}\right)\right\}^{m / 2}\|\psi\|_{\gamma, \beta} .
$$

Taking $s=1$ and $\psi \in D_{\gamma, \infty}$ the sum in Eq. (2.18) converges in the norm $\|\cdot\|_{\gamma, \alpha}$ for all $\alpha$ to a limit $B_{t} \psi \in D_{\gamma, \infty}$ such that

$$
\begin{aligned}
\left\|B_{t} \psi\right\|_{\gamma, \alpha} & \leqq \sum_{m=0}^{\infty} \frac{(2 m+1) m^{m / 2}}{m !}\left\{\sqrt{2} c_{3} t e^{\alpha+3 c t+1}\right\}^{m}\|\psi\|_{\gamma, \alpha+c t+1} \\
& \leqq c_{4} \exp \left\{c_{4} t^{2} e^{2 \alpha+6 c t}\right\}\|\psi\|_{\gamma, \alpha+c t+1}
\end{aligned}
$$

by Stirling's formula. The proof that $B_{t}$ is a semigroup is the same as in Lemma 2.1.

If $0<t<\frac{1}{4 c}$ and $\psi \in D_{\gamma, \infty}$ then taking $s=c t$ we obtain from Eq. (2.21) the alternative estimate

$$
\begin{aligned}
\left\|B_{t} \psi\right\|_{\gamma, \alpha} & \\
& \leqq \sum_{m=0}^{\infty} \frac{(2 m+1) m^{m / 2}}{m !}\left\{(2 c)^{-1 / 2} c_{3} e^{\alpha+1} t^{1 / 2}\right\}^{m}\|\psi\|_{\gamma, \alpha+2 c t} \\
& \leqq b_{0}(\alpha)\|\psi\|_{\gamma, \alpha+2 c t}
\end{aligned}
$$

where $b_{0}(\alpha)$ is a constant increasing rapidly with $\alpha$.

Having constructed $B_{t}$ as a semigroup on $\mathscr{D}$ we now compute its infinitesimal generator and obtain some associated estimates. It is easy to show that there exists a constant $a_{1}$ such that if $\psi \in D_{\gamma+2}^{(n)}$ and $t \geqq 0$ then

$$
\left\|e^{-i H_{0} t} \psi-\psi\right\|_{\gamma}^{(n)} \leqq a_{1} n t\|\psi\|_{\gamma+1}^{(n)}
$$

and

$$
\left\|e^{-i H_{0} t} \psi-\psi+i t H_{0} \psi\right\|_{\gamma}^{(n)} \leqq a_{1} n^{2} t^{2}\|\psi\|_{\gamma+2}^{(n)} \text {. }
$$


Lemma 2.3. Every $\psi \in \mathscr{D}$ lies in the domain of the infinitesimalgenerator of the semigroup $C_{t}$ on $\mathscr{D}$ and

$$
\lim _{t \rightarrow 0+} t^{-1}\left(C_{t} \psi-\psi\right)=\left(-i H_{0}-\frac{1}{2} R\right) \psi
$$

the limit being taken in $\mathscr{D}$ with its given topology. estimate

Proof. If $\psi \in D_{\gamma+2, \alpha+1}$ and $0 \leqq t \leqq \frac{1}{4 c}$ then from Eq. (2.9) we have the

Therefore

$$
\left\|C_{t} \psi-\psi\right\|_{\gamma}^{(n)} \leqq\left\|e^{-i H_{0} t} \psi-\psi\right\|_{\gamma}^{(n)}+\sum_{m=1}^{\infty} \frac{(c n t)^{m}}{m !}\|\psi\|_{\gamma}^{(n)}
$$

$$
\begin{aligned}
\| C_{t} \psi- & \psi \|_{\gamma, \alpha} \\
& \leqq a_{1} t\|\psi\|_{\gamma+1, \alpha+1} \sup _{n}\left\{n e^{-n}\right\}+c t\|\psi\|_{\gamma, \alpha+1} \sup _{n}\left\{n e^{-3 n / 4}\right\} \\
& \leqq a_{2} t\|\psi\|_{\gamma+1, \alpha+1} .
\end{aligned}
$$

\section{Secondly}

$$
\begin{aligned}
\left\|C_{t}^{(1)} \psi+\frac{1}{2} t R \psi\right\|_{\gamma}^{(n)}=\left\|\frac{1}{2} \int_{s=0}^{t}\left\{e^{-i H_{0}(t-s)} R e^{-i H_{0} s}-R\right\} \psi d s\right\|_{\gamma}^{(n)} \\
\leqq \frac{1}{2}\left\|\int_{s=0}^{t}\left(e^{-i H_{0}(t-s)}-1\right) R \psi d s\right\|_{\gamma}^{(n)} \\
\quad+\frac{1}{2}\left\|\int_{s=0}^{t} e^{-i H_{0}(t-s)} R\left(e^{-i H_{0} s}-1\right) \psi d s\right\|_{\gamma}^{(n)} \\
\leqq \\
= \\
=2 a_{1} c a_{1} n t 2 c n\|\psi\|_{\gamma}^{2}\|\psi\|_{\gamma+1}^{(n)}+\frac{1}{2} t 2 c n a_{1} n t\|\psi\|_{\gamma+1}^{(n)}
\end{aligned}
$$

Putting all these estimates into Eq. (2.7) gives

$$
\begin{aligned}
\left\|C_{t} \psi-\psi+i t H_{0} \psi+\frac{1}{2} t R \psi\right\|_{\gamma}^{(n)} \leqq & a_{1} n^{2} t^{2}\|\psi\|_{\gamma+2}^{(n)} \\
& +2 a_{1} c n^{2} t^{2}\|\psi\|_{\gamma+1}^{(n)}+\sum_{m=2}^{\infty} \frac{(c n t)^{m}}{m !}\|\psi\|_{\gamma}^{(n)} \\
\leqq & a_{3} t^{2}\|\psi\|_{\gamma+2}^{(n)}\left(n^{2}+n^{2} e^{n / 4}\right)
\end{aligned}
$$

from which we obtain

$$
\left\|C_{t} \psi-\psi+t\left(i H_{0}+\frac{1}{2} R\right) \psi\right\|_{\gamma, \alpha} \leqq a_{4} t^{2}\|\psi\|_{\gamma+2, \alpha+1} .
$$


Lemma 2.4. Every $\psi \in \mathscr{D}$ lies in the domain of the infinitesimalgenerator of the semigroup $B_{t}$ on $\mathscr{D}$ and

$$
\lim _{t \rightarrow 0+} t^{-1}\left(B_{t} \psi-\psi\right)=Z \psi
$$

the limit being taken in the topology of $\mathscr{D}$.

Proof. If $\psi \in D_{\gamma+2, \alpha+3}$ and $0<t<\frac{1}{4 c}$, then defining $s=1$, Eq. (2.21) becomes

$$
\left\|B_{t}^{(m)} \psi\right\|_{\gamma, \alpha} \leqq \frac{(2 m+1) m^{m / 2}}{m !}\left\{\sqrt{2} c_{3} t e^{\alpha+2}\right\}^{m}\|\psi\|_{\gamma, \alpha+2} .
$$

Eqs. (2.18), (2.27), and (2.31) now yield

$$
\begin{aligned}
& \left\|B_{t} \psi-\psi\right\|_{\gamma, \alpha} \\
& \quad \leqq a_{2} t\|\psi\|_{\gamma+1, \alpha+1}+\sum_{m=1}^{\infty} \frac{(2 m+1) m^{m / 2}}{m !}\left\{\sqrt{2} c_{3} t e^{\alpha+2}\right\}^{m}\|\psi\|_{\gamma, \alpha+2} \\
& \quad \leqq b_{1}(\alpha) t\|\psi\|_{\gamma+1, \alpha+2} .
\end{aligned}
$$

Eqs. (2.19), (2.11), (2.17), and (2.27) yield

$$
\begin{aligned}
& \left\|B_{t}^{(1)} \psi+i t H_{1} \psi\right\|_{\gamma, \alpha} \\
& \quad \leqq\left\|\int_{s=0}^{t}\left(C_{t-s}-1\right) H_{1} \psi d s\right\|_{\gamma, \alpha}+\left\|\int_{s=0}^{t} C_{t-s} H_{1}\left(C_{s}-1\right) \psi d s\right\|_{\gamma, \alpha} \\
& \quad \leqq b_{2}(\alpha) t^{2}\|\psi\|_{\gamma+1, \alpha+3} .
\end{aligned}
$$

Eqs. (2.18), (2.29), (2.31), and (2.33) now yield

$$
\left\|B_{t} \psi-\psi-t Z \psi\right\|_{\gamma, \alpha} \leqq b_{3}(\alpha) t^{2}\|\psi\|_{\gamma+2, \alpha+3} .
$$

This estimate yields the statement of the lemma.

Lemma 2.5. The semigroup $B_{t}$ on $\mathscr{D}$ has a unique extension to a strongly continuous contraction semigroup on $\mathscr{F}$ whose infinitesimal generator is an extension of $Z$ defined on $\mathscr{D}$.

Proof. We take $\gamma=\gamma_{0}, \alpha=\alpha_{0}$, and $\psi \in \mathscr{D}$.

If $t, t^{\prime} \geqq 0$ and $0<t^{\prime}-t<\frac{1}{4 c}$, then by Eqs. (2.22) and (2.32)

$$
\begin{aligned}
&\left\|B_{t^{\prime}} \psi-B_{t} \psi\right\| \\
& \leqq c_{0}\left\|\left(B_{t^{\prime}-t}-1\right) B_{t} \psi\right\|_{\gamma, \alpha} \\
& \leqq c_{0} b_{1}\left(t^{\prime}-t\right) c_{4} \exp \left\{c_{4} t^{2} e^{2 \alpha+4+6 c t}\right\}\|\psi\|_{\gamma+1, \alpha+c t+3} .
\end{aligned}
$$


This establishes that the map $t \rightarrow B_{t} \psi \in \mathscr{F}$ is norm continuous for all $\psi \in \mathscr{D}$. By Lemma 2.4

$$
\begin{aligned}
& \lim _{t \rightarrow 0+} t^{-1}\left\{\left\langle B_{t} \psi, B_{t} \psi\right\rangle-\langle\psi, \psi\rangle\right\} \\
&=\lim _{t \rightarrow 0+} t^{-1}\left\langle B_{t} \psi-\psi, B_{t} \psi\right\rangle+\lim _{t \rightarrow 0+} t^{-1}\left\langle\psi, B_{t} \psi-\psi\right\rangle \\
&=\langle Z \psi, \psi\rangle+\langle\psi, Z \psi\rangle \\
&=-\langle R \psi, \psi\rangle \leqq 0 .
\end{aligned}
$$

Therefore $t \rightarrow\left\|B_{t} \psi\right\|^{2}$ is continuous and differentiable on the right with negative derivative for all $t \geqq 0$, and so is a monotone decreasing function. It follows that $B_{t}$ is a contraction on the dense subspace $\mathscr{D}$ of $\mathscr{F}$ and has a unique extension to a contraction on $\mathscr{F}$. The remaining statements of the lemma now follow by elementary arguments.

From now on we shall use $B_{t}$ to denote the contraction semigroup on $\mathscr{F}$ defined by the above lemma. As in [1] we define the strongly continuous contraction semigroup $S_{t}$ on $V=\mathscr{T}_{s}(\mathscr{F})$ by $S_{t}(\varrho)=B_{t} \varrho B_{t}^{*}$ for all $\varrho \in V$.

Lemma 2.6. Let $R^{\prime}$ be a bounded operator on $\mathscr{F}$ with $0 \leqq R^{\prime} \leqq R$ as sesquilinear forms on $\mathscr{D} \times \mathscr{D}$. Then for all $\varrho \in V^{+}$

$$
\lim _{t \rightarrow 0+} \sup \operatorname{tr}\left[t^{-1}\left(S_{t} \varrho-\varrho\right)\right]+\operatorname{tr}\left[R^{\prime} \varrho\right] \leqq 0 .
$$

Proof. If $\psi \in \mathscr{D}$ then $B_{t} \psi \in \mathscr{D}$ for all $t \geqq 0$ and

$$
\frac{d}{d t}\left\langle B_{t} \psi, B_{t} \psi\right\rangle+\left\langle R^{\prime} B_{t} \psi, B_{t} \psi\right\rangle \leqq 0
$$

so

$$
\left\langle B_{t} \psi, B_{t} \psi\right\rangle+\int_{s=0}^{t}\left\langle R^{\prime} B_{s} \psi, B_{s} \psi\right\rangle d s \leqq 0 .
$$

This establishes that

$$
\operatorname{tr}\left[S_{t} \varrho\right]+\int_{s=0}^{t} \operatorname{tr}\left[R^{\prime} S_{s}(\varrho)\right] d s \leqq 0
$$

first for all $\varrho=\psi \otimes \bar{\psi}$ where $\psi \in \mathscr{D}$ and then by continuity arguments for all $\varrho \in V^{+}$. The required result follows by differentiating this equation at $t=0$.

\section{§ 3. Construction and Convergence of the Approximate Sub-Stochastic Processes}

We turn now to the rigorous construction of the bounded stochastic kernels $\mathscr{J}^{\delta}$ where $0<\delta<1 / 2$. Throughout this section we shall take $\alpha=\alpha_{0}$ and $\gamma=\gamma_{0} . V_{0}$ will denote the dense subspace of $V$ consisting 
of operators of finite rank which can be written in the form $\varrho=\sum_{r=1}^{n} t_{r} \psi_{r} \otimes \bar{\psi}_{r}$ where $\psi_{r} \in \mathscr{D}$ for $1 \leqq r \leqq n$.

First note that if $\psi \in \mathscr{D}$ then

$$
\begin{aligned}
\| a(k) e^{-\delta N} & \psi \\
& \leqq c_{0}\left\|a(k) e^{-\delta N} \psi\right\|_{\gamma, \alpha} \\
& \leqq c_{0} \sup _{n}\left\{e^{-\delta n} e^{n \alpha} \sqrt{n+1} m_{0}^{\gamma} \omega(k)^{-\gamma}\|\psi\|_{\gamma}^{(n+1)}\right\} \\
& \leqq c_{5} \omega(k)^{-\gamma} \delta^{-1 / 2}\|\psi\|_{\gamma, \alpha} .
\end{aligned}
$$

From this estimate and Eq. (1.17) it is immediate that for all $\psi \in \mathscr{D}$ the integral

$$
\begin{aligned}
& \mathscr{J}(E, \delta, \varphi, \psi) \\
& =\frac{1}{2}(2 \pi)^{-3} \iiint \frac{\mu(x) e^{-i x \cdot(k-h)}}{\omega(k)^{1 / 2} \omega(h)^{1 / 2}} \chi_{E}(x)\left\{a(k) e^{-\delta N} \varphi\right\} \\
& \quad \otimes\left\{a(h) e^{-\delta N} \psi\right\}^{-} d k d h d x
\end{aligned}
$$

defines an element of $\mathscr{T}(\mathscr{F})$. Compare Eq. (1.6).

Theorem 3.1. There exists a unique bounded stochastic kernel $\mathscr{J}^{\delta}$ on $X, V$ for all $\delta>0$ such that for all $\psi \in \mathscr{D}$ and $E \leqq X$

$$
\mathscr{J}^{\delta}(E, \psi \otimes \bar{\psi})=\mathscr{J}(E, \delta, \psi, \psi) \text {. }
$$

The interaction rate $R^{\delta}$ of $\mathscr{J}^{\delta}$ is the bounded positive operator $R^{\delta}=e^{-2 \delta N} R$ on $\mathscr{F}$.

Proof. Since $\mathscr{J}(E, \delta, \cdot, \cdot): \mathscr{D} \times \mathscr{D} \rightarrow \mathscr{T}(\mathscr{F})$ is sesquilinear it induces a positive linear map $\mathscr{J}^{\delta}(E, \cdot): V_{0} \rightarrow V$ such that Eq. (3.3) holds. If

then

$$
\varrho=\sum_{r=1}^{n} t_{r} \psi_{r} \otimes \bar{\psi}_{r} \in V_{0}
$$

$\operatorname{tr}\left[\mathscr{J}^{\delta}(X, \varrho)\right]$

$=\sum_{r=1}^{n} t_{r} \operatorname{tr}\left[\mathscr{J}\left(X, \delta, \psi_{r}, \psi_{r}\right)\right]$

$=\sum_{r=1}^{n} t_{r} \frac{1}{2}(2 \pi)^{-3} \iiint \frac{\mu(x) e^{-i x \cdot(k-h)}}{\omega(k)^{1 / 2} \omega(h)^{1 / 2}}\left\langle a(k) e^{-\delta N} \psi_{r}, a(h) e^{-\delta N} \psi_{r}\right\rangle d x d k d h$

$=\sum_{r=1}^{n} t_{r}\left\langle R^{\delta} \psi_{r}, \psi_{r}\right\rangle$

$=\operatorname{tr}\left[R^{\delta} \varrho\right]$ 
where $R^{\delta}$ is the bounded positive operator of Eq. (1.13). Now if $E \subseteq X$ and $\varrho \in V_{0}$ there exist $\varrho_{+}, \varrho_{-} \in V_{0}$ with $\varrho=\varrho_{+}-\varrho_{-}$and $\|\varrho\|=\left\|\varrho_{+}\right\|+\left\|\varrho_{-}\right\|$. Therefore

$$
\begin{aligned}
\left\|\mathscr{J}^{\delta}(E, \varrho)\right\| & \leqq\left\|\mathscr{J}^{\delta}\left(E, \varrho_{+}\right)\right\|+\left\|\mathscr{J}^{\delta}\left(E, \varrho_{-}\right)\right\| \\
& =\operatorname{tr}\left[\mathscr{J}^{\delta}\left(E, \varrho_{+}\right)\right]+\operatorname{tr}\left[\mathscr{J}^{\delta}\left(E, \varrho_{-}\right)\right] \\
& \leqq \operatorname{tr}\left[\mathscr{J}^{\delta}\left(X, \varrho_{+}\right)\right]+\operatorname{tr}\left[\mathscr{J}^{\delta}\left(X, \varrho_{-}\right)\right] \\
& \leqq\left\|R^{\delta}\right\| \operatorname{tr}\left[\varrho_{+}\right]+\left\|R^{\delta}\right\| \operatorname{tr}\left[\varrho_{-}\right] \\
& =\left\|R^{\delta}\right\|\|\varrho\| .
\end{aligned}
$$

Therefore $\mathscr{J}^{\delta}(E, \cdot)$ is a bounded positive linear map defined on the dense subspace $V_{0}$ of $V$, and has a unique extension to a bounded positive linear map from $V$ to $V$. Since $E \rightarrow \mathscr{J}^{\delta}(E, \varrho)$ is clearly $\sigma$-additive for all $\varrho \in V_{0}$ this is also true, by taking limits, for all $\varrho \in V$, so $\mathscr{J}^{\delta}$ is a bounded stochastic kernel.

Theorem 3.2. There exists a sub-stochastic process $\mathscr{E}^{\delta}$ on $X, V$ whose infinitesimal generators are $Z, \mathscr{J}^{\delta}$.

Proof. $\mathscr{E} \delta$ is constructed exactly as in Theorem 4.7 of [1], except that Eq. (4.15) of [1] is replaced by Eq. (2.37) applied to the case $R^{\prime}=R^{\delta}$. This then gives

$$
\limsup _{t \rightarrow 0+} \operatorname{tr}\left[\frac{1}{t}\left(T_{t} \varrho-\varrho\right)\right] \leqq 0
$$

for all $\varrho \in V^{+}$which proves that $\mathscr{E}^{\delta}$ is substochastic.

The following estimates will be needed in the proof of the main theorem of the section.

Lemma 3.3. Let $0<t_{1}<t_{2}<\cdots<t_{n} \leqq t \leqq \frac{1}{4 c}$, let $\delta \geqq 0$ and let $\psi \in \mathscr{D}$. Define

$$
\begin{gathered}
G_{n}^{\delta}\left(t_{1} \ldots t_{n}, k_{1} \ldots k_{n}\right) \\
=\left(B_{t-t_{n}} a\left(k_{n}\right) e^{-\delta N} B_{t_{n}-t_{n-1}} a\left(k_{n-1}\right) e^{-\delta N} \ldots a\left(k_{1}\right) e^{-\delta N} B_{t} \psi .\right.
\end{gathered}
$$

Then

$$
\begin{gathered}
\left\|G_{n}^{\delta} \psi\right\|_{\gamma, \alpha} \leqq b_{4}^{n}(\alpha) n^{n / 2} \omega\left(k_{1}\right)^{-\gamma} \ldots \omega\left(k_{n}\right)^{-\gamma}\|\psi\|_{\gamma, \alpha+2}, \\
\left\|G_{n}^{\delta} \psi-G_{n}^{0} \psi\right\|_{\gamma, \alpha} \leqq \delta n b_{4}^{n}(\alpha) n^{n / 2} \omega\left(k_{1}\right)^{-\gamma} \ldots \omega\left(k_{n}\right)^{-\gamma}\|\psi\|_{\gamma, \alpha+3} .
\end{gathered}
$$

Proof. Eq. (3.8) is a consequence of Eq. (2.23) with the extra estimate

$$
\|a(k) \psi\|_{\gamma, \alpha} \leqq m_{0}^{\gamma} \omega(k)^{-\gamma} \sqrt{n}\|\psi\|_{\gamma, \alpha+\frac{1}{n}} .
$$


Eq. (3.9) is obtained by using the additional estimate

and writing

$$
\left\|e^{-\delta N} \psi-\psi\right\|_{\gamma, \alpha} \leqq \delta\|\psi\|_{\gamma, \alpha+1}
$$

where

$$
G_{n}^{\delta} \psi-G_{n}^{0} \psi=\sum_{r=1}^{n} \psi_{r}
$$

$$
\psi_{r}=B_{t-t_{n}} a\left(k_{n}\right) e^{-\delta N} \ldots
$$$$
\ldots a\left(k_{r+1}\right) e^{-\delta N} B_{t_{r+1}-t_{r}} a\left(k_{r}\right)\left(e^{-\delta N}-1\right) B_{t_{r}-t_{r-1}} a\left(k_{r-1}\right) B_{t_{r-1}-t_{r-2}} \ldots
$$

$$
\ldots a\left(k_{1}\right) B_{t_{1}} \psi \text {. }
$$

Lemma 3.4. If $F \cong A_{t}^{n}$ and $\psi \in \mathscr{D}$ and $0<t<\frac{1}{4 c}$ and $\delta>0$ then $\mathscr{E}_{t}^{\delta}(F, \psi \otimes \bar{\psi})$

$$
\begin{aligned}
= & \frac{1}{2 n}(2 \pi)^{-3 n} \iiint \int \prod_{r=1}^{n} \frac{\mu\left(x_{r}\right) e^{-i x_{r} \cdot\left(k_{r}-h_{r}\right)}}{\omega\left(k_{r}\right)^{1 / 2} \omega\left(h_{r}\right)^{1 / 2}} \chi_{F}\left(x_{1} \ldots x_{n}, t_{1} \ldots t_{n}\right) \ldots \\
& \ldots\left\{G_{n}^{\delta}\left(t_{1} \ldots t_{n}, k_{1} \ldots k_{n}\right) \psi\right\} \otimes\left\{G_{n}^{\delta}\left(t_{1} \ldots t_{n}, h_{1} \ldots h_{n}\right)\right\}^{-} \\
& \prod_{r=1}^{n} d t_{r} d x_{r} d k_{r} d h_{r} .
\end{aligned}
$$

This integral also exists for $\delta=0$ as an element of $V$ and

$$
\left\|\mathscr{E}_{t}^{\mathscr{\delta}}(F, \psi \otimes \bar{\psi})-\mathscr{E}_{t}^{0}(F, \psi \otimes \psi)\right\| \leqq \delta n b^{n}(\alpha) t^{n}\|\psi\|_{\gamma, \alpha+3}^{2}
$$

where $b(\alpha)$ is a constant independent of $F$.

Proof. The convergence of the integral for $\delta \geqq 0$ follows from Eq. (3.8). That the integral does give $\mathscr{E}_{t}^{\delta}(F, \psi \otimes \bar{\psi})$ if $\delta>0$ follows from Eq. (4.13) of [1] extended to arbitrary Borel sets $F \cong A_{t}^{n}$. Eq. (3.9) together with the observation that

$F \leqq\left\{\left(x_{1}, t_{1}, \ldots, x_{n}, t_{n}\right): 0<t_{1}<\cdots<t_{n} \leqq t \quad\right.$ and $\left.\quad x_{1}, \ldots, x_{n} \in X\right\}$ gives

$$
\begin{gathered}
\left\|\mathscr{E}_{t}^{\delta}(F, \psi \otimes \bar{\psi})-\mathscr{E}_{t}^{0}(F, \psi \otimes \bar{\psi})\right\| \\
\leqq 2 \delta n b_{4}^{2 n} n^{n}\|\psi\|_{\gamma, \alpha+3}^{2}\left\{\int \omega(k)^{-\gamma} d k\right\}^{2 n}\left\{\int \mu(x) d x\right\}^{n} \frac{t^{n}}{n !}
\end{gathered}
$$

which yields Eq. (3.15) by use of Stirling's formula.

Lemma 3.5. Let $(Y, d y)$ be a measure space and $\psi: Y \rightarrow \mathscr{D}$ a measurable function such that

$$
\int_{Y}\left\|\psi_{y}\right\|_{\gamma+2, \alpha+3}^{2} d y<\infty .
$$


Then the equation

$$
\varrho=\int_{Y} \psi_{y} \otimes \bar{\psi}_{y} d y
$$

defines an element $\varrho \in V^{+}$and if $0<t<\frac{1}{4 c}$

$$
\left|\operatorname{tr}\left[S_{t} \varrho-\varrho\right]+t \int_{t}\left\langle R \psi_{y}, \psi_{y}\right\rangle d y\right| \leqq b_{5} t^{2} \int\left\|\psi_{y}\right\|_{\gamma+2, \alpha+3}^{2} d y .
$$

If $0<t<\frac{1}{4 c}$ the equation

$$
\varrho_{t}=\int \mathscr{E}_{t}^{0}\left(A_{t}^{1}, \psi_{y} \otimes \bar{\psi}_{y}\right) d y
$$

defines an element of $V^{+}$and

$$
\left|\operatorname{tr}\left[\varrho_{t}\right]-t \int_{Y}\left\langle R \psi_{y}, \psi_{y}\right\rangle d y\right| \leqq b_{6} t^{2} \int\left\|\psi_{y}\right\|_{\gamma+1, \alpha+3}^{2} d y .
$$

Proof. That $\varrho \in V^{+}$follows from Eqs. (1.20) and (1.21). By Eqs. (2.32) and (2.34)

$$
\begin{aligned}
& \left|\operatorname{tr}\left[S_{t} \varrho-\varrho\right]+t \int_{Y}\left\langle R \psi_{y}, \psi_{y}\right\rangle d y\right| \\
\leqq & \left.\int\left\langle B_{t} \psi_{y}-\psi_{y}+t Z \psi_{y}, \psi_{y}\right\rangle+\left\langle\psi_{y}, B_{t} \psi_{y}-\psi_{y}+t Z \psi_{y}\right\rangle+\left\langle B_{t} \psi_{y}-\psi_{y}, B_{t} \psi_{y}-\psi_{y}\right\rangle \mid d\right] \\
\leqq & \left(2 b_{3} t^{2}+b_{1}^{2} t^{2}\right) \int\left\|\psi_{y}\right\|_{\gamma+2, \alpha+3}^{2} d y
\end{aligned}
$$

which is Eq. (3.19). From Eq. (3.14) we obtain

$$
\left\|\mathscr{E}_{t}^{0}\left(A_{t}^{1}, \psi_{y} \otimes \bar{\psi}_{y}\right)\right\| \leqq b_{7}\left\|\psi_{y}\right\|_{\gamma, \alpha+2}^{2}
$$

which together with Eq. (3.18) shows that $\varrho_{t}$ is well defined as an element of $V^{+}$. Now

$$
\begin{aligned}
& \operatorname{tr}\left[\mathscr{E}_{t}^{0}\left(A_{t}^{1}, \psi_{y} \otimes \bar{\psi}_{y}\right)\right] \\
& =\frac{1}{2}(2 \pi)^{-3} \iiint \int_{0<s<t} \frac{\mu(x) e^{-i x \cdot(k-h)}}{\omega(k)^{1 / 2} \omega(h)^{1 / 2}}\left\langle B_{t-s} a(k) B_{s} \psi_{y}, B_{t-s} a(h) B_{s} \psi_{y}\right\rangle \ldots \\
& \quad \ldots d x d s d k d h .
\end{aligned}
$$

Comparing this with Eq. (1.9) and using Eqs. (2.23), (2.32), (3.10) to estimate the difference yields Eq. (3.21).

Theorem 3.6. There exists a quantum stochastic process $\mathscr{E}_{t}$ on $X, V$ and a number $t_{0}>0$ such that if $\varrho \in V, 0 \leqq t \leqq t_{0}$ and $F \leqq X_{t}$ then

$$
\lim _{\delta \rightarrow 0}\left\|\mathscr{E}_{t}^{\delta}(F, \varrho)-\mathscr{E}_{t}(F, \varrho)\right\|=0
$$

the convergence being uniform with respect to such $t, F$. 
Proof. We define $t_{0}=\min \left\{\frac{1}{4 c}, \frac{1}{2 b}\right\}$. If $\varrho=\sum_{r=1}^{m} t_{r} \psi_{r} \otimes \bar{\psi}_{r} \in V_{0}$ and $0<\delta, \delta^{\prime} \leqq \delta_{0}$ then by Eq. (3.15)

$$
\begin{aligned}
& \left\|\mathscr{E}_{t}^{\delta}(F, \varrho)-\mathscr{E}_{t}^{\delta^{\prime}}(F, \varrho)\right\| \\
& \leqq \sum_{n=1}^{\infty}\left\|\mathscr{E}_{t}^{\delta}\left(F \cap A_{t}^{n}, \varrho\right)-\mathscr{E}_{t}^{\delta^{\prime}}\left(F \cap A_{t}^{n}, \varrho\right)\right\| \\
& \leqq \sum_{r=1}^{m} \sum_{n=1}^{\infty} 2 \delta_{0} n b^{n} t_{0}^{n} t_{r}\left\|\psi_{r}\right\|_{\gamma, \alpha+3}^{2} \\
& \leqq \delta_{0} \sum_{r=1}^{m} \sum_{n=1}^{\infty} 2 n 2^{-n} t_{r}\|\psi\|_{\gamma, \alpha+3}^{2} \\
& \leqq K \delta_{0}
\end{aligned}
$$

so for such $\varrho$ the limit does exist uniformly. Since $V_{0}$ is dense in $V$ and each $\mathscr{E}_{t}^{\delta}(F, \cdot): V \rightarrow V$ is a contraction the limit exists uniformly for all $\varrho \in V$. We have also shown that if $\psi \in \mathscr{D}$ and $F \cong A_{t}^{n}$ then

$$
\mathscr{E}_{t}(F, \psi \otimes \bar{\psi})=\mathscr{E}_{t}^{0}(F, \psi \otimes \bar{\psi}) .
$$

From its definition as a limit it is easy to verify that $\mathscr{E}_{t}$ has the following properties, subject to the condition that $0 \leqq t \leqq t_{0}$.

(i) $\varrho \rightarrow \mathscr{E}_{t}(F, \varrho)$ is positive linear on $V$ for all $F \cong X_{t}$;

(ii) $F \rightarrow \mathscr{E}_{t}(F, \varrho)$ is $\sigma$-additive for all $\varrho \in V$;

(iii) $\operatorname{tr}\left[\mathscr{E}_{t}\left(X_{t}, \varrho\right)\right] \leqq \operatorname{tr}[\varrho]$ if $\varrho \in V^{+}$;

(iv) $t \rightarrow \mathscr{E}_{t}\left(X_{t}, \varrho\right)$ is continuous for all $\varrho \in V$;

(v) $\mathscr{E}_{s+t}=\mathscr{E}_{s} \circ \mathscr{E}_{t}$ in the sense of composition defined in [3] provided $0 \leqq s, t$ and $s+t \leqq t_{0}$.

If $t \geqq 0$ is arbitrary and $N$ is the integer such that $(N-1) t_{0}<t \leqq N t_{0}$ we define $\mathscr{E}_{t}$ as the composition

$$
\mathscr{E}_{t}=\left(\mathscr{E}_{t_{0}}\right)^{N-1} \circ \mathscr{E}_{t-(N-1) t_{0}}
$$

From the above properties it is immediate that $\mathscr{E}_{t}$ is a sub-stochastic process on $X, V$ and the only remaining problem is to prove that $\mathscr{E}_{t}$ is actually stochastic.

If $\psi \in \mathscr{D}$ and $0 \leqq t_{1} \leqq t_{0}$ then $\mathscr{E}_{t_{1}}\left(X_{t}, \psi \otimes \bar{\psi}\right)$ is of the form

where

$$
\varrho=\int_{Y} \psi_{y} \otimes \bar{\psi}_{y} d y
$$

5 Commun. math. Phys., Vol. 22

$$
\int_{Y}\left\|\psi_{y}\right\|_{\gamma+2, \alpha+3}^{2} d y<\infty
$$


by Eqs. (3.25), (3.14), and (3.8). By Lemma 3.5

$$
\lim _{t \rightarrow 0+} \operatorname{tr}\left[t^{-1}\left(\mathscr{E}_{t}\left(\{z\} \cup A_{t}^{1}, \varrho\right)-\varrho\right)\right]=0
$$

so

$$
\liminf _{t \rightarrow 0+} \operatorname{tr}\left[t^{-1}\left(\mathscr{E}_{t}\left(X_{t}, \varrho\right)-\varrho\right)\right] \geqq 0
$$

which proves that the continuous function

$$
t \rightarrow \operatorname{tr}\left[\mathscr{E}_{t}\left(X_{t}, \psi \otimes \bar{\psi}\right)\right]
$$

is monotone increasing for $0 \leqq t \leqq t_{0}$. Since the convex combinations of such states $\psi \otimes \bar{\psi}$ are dense in $V^{+}$and $\mathscr{E}_{t}$ is known to be sub-stochastic it follows that

$$
\operatorname{tr}\left[\mathscr{E}_{t}\left(X_{t}, \varrho\right)\right]=\operatorname{tr}[\varrho]
$$

for all $\varrho \in V^{+}$and all $0 \leqq t \leqq t_{0}$. It is now immediate that this also holds for all $\varrho \in V$ and all $t \geqq 0$.

Before closing the section we note that all the calculations we have done can be performed if $X$ is an arbitrary locally compact abelian group, the crucial starting estimates being Eqs. (1.17), (2.3), (2.4), (2.15), and (2.16). In particular if we impose periodic boundary conditions by taking $X=\Pi^{3}$ a translation invariant process is obtained if $\mu$ and $j$ are both taken to be constant on $X$.

\section{§ 4. The Harmonic Oscillator with External Damping}

If we take $X$ to consist of just one point $e$ then we have a model of a displaced harmonic oscillator interacting with an absorptive particle detector. It will become apparent that this is closely related to a method of quantizing a certain ordinary differential equation, which however cannot be realized by a one-parameter unitary group on the appropriate Hilbert space. We define

$$
\mathscr{H}=\left\{\left\{\psi_{n}\right\}_{n=0}^{\infty}: \psi_{n} \in \mathbb{C} \text { and }\|\psi\|=\left\{\sum_{n=0}^{\infty}\left|\psi_{n}\right|^{2}\right\}^{1 / 2}<\infty\right\}
$$

on which we need only the one-parameter family of norms

$$
\|\psi\|_{\alpha}=\sup _{n}\left\{\left|\psi_{n}\right| e^{n \alpha}\right\}
$$

to define the domain $\mathscr{D}=\bigcap_{\alpha>0} D_{\alpha}$. To fix notation we let $\omega, \mu$ be two positive numbers, $z_{0}$ an arbitrary complex number and $a^{*}, a$ the creation and annihilation operators respectively on $\mathscr{H}$. The process $\mathscr{E}$ is constructed on $V=\mathscr{T}_{s}(\mathscr{H})$ by the methods of Section 3 using the formal 
definitions

$$
H=\omega a^{*} a-\frac{1}{\sqrt{2 \omega}}\left(\bar{z}_{0} a+z_{0} a^{*}\right)
$$

and

$$
\mathscr{J}(e, \varrho)=\mu a \varrho a^{*}
$$

so

$$
Z=-i H-\frac{1}{2} \mu a^{*} a .
$$

We define

$$
T_{t}(\varrho)=\mathscr{E}_{t}\left(X_{t}, \varrho\right)
$$

where

$$
X_{t}=\left\{\left(t_{1}, \ldots, t_{n}\right): 0<t_{1}<\cdots<t_{n} \leqq t \quad \text { and } \quad 0 \leqq n<\infty\right\}
$$

so that $T_{t}$ is a strongly continuous one-parameter semigroup on $V=\mathscr{T}_{s}(\mathscr{H})$ such that if $\varrho \in V^{+}$then

$$
T_{t}(\varrho) \in V^{+} \text {and } \operatorname{tr}\left[T_{t}(\varrho)\right]=\operatorname{tr}[\varrho] .
$$

For the remainder of the section we investigate the properties of $T_{t}$ alone, rather than the entire stochastic process $\mathscr{E}_{t}$, and we do this by introducing the family of coherent states [4]. For every $z \in \mathbb{C} \psi(z)$ is the unit vector in $\mathscr{H}$ such that

$$
\psi_{n}(z)=e^{-\frac{1}{2}|z|^{2}} \frac{z^{n}}{\sqrt{n !}} .
$$

Each $\psi(z)$ lies in $\mathscr{D}$ and is an eigenvector of the annihilation operator with

$$
a\{\psi(z)\}=z \psi(z) .
$$

States of the form $\varrho=\psi(z) \otimes \overline{\psi(z)}$ are called pure coherent states [4]. The following theorem shows that these states have very special properties with respect to the semigroup $T_{t}$.

Theorem 4.1. If $\varrho \in V^{+}$is not a pure coherent state then $T_{t}(\varrho)$ is not a pure state provided $t>0$ is small enough. If $\varrho$ is a pure coherent state then $T_{t}(\varrho)$ is a pure coherent state for all $t \geqq 0$.

Proof. Because the set of pure states in $V^{+}$is norm closed and $T_{t}$ is strongly continuous, $T_{t}(\varrho)$ can only be a pure state for arbitrarily small $t>0$ if $\varrho$ is already pure. We now suppose $\varrho=\psi \otimes \bar{\psi}$ where $\psi \in \mathscr{H}$ and that $T_{t}(\varrho)$ is pure for all $0<t \leqq 2 t_{0}$.

Since

$$
\left(C_{t} \psi\right)_{n}=e^{\left(-i \omega-\frac{1}{2} \mu\right) n t} \psi_{n}
$$


we have the estimate

$$
\left\|C_{t} \psi\right\|^{(n)} \leqq e^{-\frac{1}{2} \mu n t}\|\psi\|^{(n)} .
$$

Using this instead of Eq. (2.10) to recalculate Lemma 2.2 we obtain an estimate of the form

$$
\left\|B_{t} \psi\right\|_{\alpha+\frac{1}{2} \mu t} \leqq K(\alpha, t)\|\psi\|_{\alpha}
$$

so $B_{t} \psi \in \operatorname{Dom}(a)$ for all $t>0$. Now if $\delta>0, \Phi=B_{t_{0}} \psi$, and $0<t \leqq t_{0}$

$$
\begin{aligned}
& T_{t_{0}+t}^{\delta}(\varrho) \geqq T_{t}^{\delta}(\Phi \otimes \bar{\Phi}) \\
& \geqq\left(B_{t} \Phi\right) \otimes\left(B_{t} \Phi\right)^{-}+\mu \int_{s=0}^{t}\left(B_{t-s} a e^{-\delta N} \Phi\right) \otimes\left(B_{t-s} a e^{-\delta N} \Phi\right)^{-} d s .
\end{aligned}
$$

Going to the limit as $\delta \rightarrow 0$ by using the fact that $\Phi \in \operatorname{Dom}(a)$ we obtain $T_{t_{0}+t}(\varrho) \geqq\left(B_{t} \Phi\right) \otimes\left(B_{t} \Phi\right)^{-}+\mu \int_{s=0}^{t}\left(B_{t-s} a \Phi\right) \otimes\left(B_{t-s} a \Phi\right)^{-} d s$.

Since the left-hand side is a pure state when $0<t \leqq t_{0}$ the right-hand side must be a pure state however small $t>0$ is, so $\Phi$ and $a \Phi$ are proportional and $\Phi$ is an eigenvector of $a$ and hence a coherent state. Now letting $t_{0} \rightarrow 0$ and using the fact that the coherent states form a norm closed subset of $V$, it follows that $\psi$ is a coherent state.

Conversely suppose $\varrho=\psi(z) \otimes \overline{\psi(z)}$ is a pure coherent state. If $z(t)$ and $\lambda(t)$ are two complex-valued functions of $t \geqq 0$ then

$$
\frac{d}{d t}\left\{\lambda(t) e^{\frac{1}{2}|z(t)|^{2}} \psi\{z(t)\}\right\}=Z\left\{\lambda(t) e^{\frac{1}{2}|z(t)|^{2}} \psi\{z(t)\}\right\}
$$

if and only if

$$
\begin{aligned}
\frac{d}{d t}\left\{\lambda(t) e^{\frac{1}{2}|z(t)|^{2}}\right\} \frac{z(t)^{n}}{\sqrt{n !}}+\lambda(t) e^{\frac{1}{2}|z(t)|^{2}} n \frac{d z}{d t} \frac{z(t)^{n-1}}{\sqrt{n !}} \\
=\lambda(t) e^{\frac{1}{2}|z(t)|^{2}}\left\{\left(-i \omega-\frac{1}{2} \mu\right) n \frac{z(t)^{n}}{\sqrt{n !}}\right. \\
\left.\quad+\frac{1}{\sqrt{2 \omega}} \sqrt{n+1} \bar{z}_{0} \frac{z(t)^{n+1}}{\sqrt{(n+1) !}}+\frac{1}{\sqrt{2 \omega}} \sqrt{n} z_{0} \frac{z(t)^{n-1}}{\sqrt{(n-1) !}}\right\}
\end{aligned}
$$

Therefore if $\lambda(t)$ and $z(t)$ are the solutions of the ordinary differential equations

$$
\begin{gathered}
\frac{d z}{d t}=\left(-i \omega-\frac{1}{2} \mu\right) z(t)+\frac{z_{0}}{\sqrt{2 \omega}}, \\
\frac{d}{d t}\left\{\lambda(t) e^{\frac{1}{2}|z(t)|^{2}}\right\}=\frac{\bar{z}_{0}}{\sqrt{2 \omega}} z(t) \lambda(t) e^{\frac{1}{2}|z(t)|^{2}}
\end{gathered}
$$


subject to the initial conditions $z(0)=z$ and $\lambda(0)=1$, we have shown quite rigorously that Eq. (4.16) is satisfied. By the theory of one-parameter semigroups it follows that

$$
B_{t}\{\psi(z)\}=\lambda(t) \psi\{z(t)\}
$$

for all $t \geqq 0$. Now by Eqs. (3.7) and (4.10) it is immediate that $G_{n}^{0} \psi(z)$ is proportional to $\psi\{z(t)\}$ for all $t \geqq 0$ and all $n$ so by Eq. (3.25)

$$
T_{t}\{\psi(z) \otimes \overline{\psi(z)}\}=\psi\{z(t)\} \otimes \psi\{z(t)\}
$$

first for all sufficiently small $t$ and hence for all $t \geqq 0$, the constant of proportionality being unity because of Eq. (4.8).

The above theorem shows that $T_{t}$ provides a quantization of the differential Eq. (4.18). Since the pure coherent states span a dense linear subspace of $V$ by [4], $T_{t}$ is unique determined by Eq. (4.21). Moreover there does not exist any unitary group $U(t)$ on $\mathscr{H}$ such that $T_{t}(\varrho)$ $=U(t) \varrho U(t)^{*}$ because $T_{t}$ does not map general pure states to pure states.

The following theorem provides an example of an infinite-dimensional quantum stochastic process with an equilibrium state. This and related properties are studied systematically in [2].

Theorem 4.2. There exists a pure coherent state $\psi\left(z_{1}\right) \otimes \overline{\psi\left(z_{1}\right)}$ where

$$
z_{1}=\frac{z_{0}}{\sqrt{2 \omega}}\left(i \omega+\frac{1}{2} \mu\right)^{-1}
$$

such that for all states $\varrho \in V$

$$
\lim _{t \rightarrow \infty} T_{t}(\varrho)=\operatorname{tr}[\varrho] \psi\left(z_{1}\right) \otimes \overline{\psi\left(z_{1}\right)}
$$

where the limit is taken in the norm topology of $V$.

Proof. For pure coherent states $\varrho$ the result is an immediate consequence of the form of the explicit solution of Eq. (4.18). Since linear combinations of pure coherent states are dense in $V$ and $T_{t}$ is a contraction semigroup, Eq. (4.23) now holds for arbitrary $\varrho \in V$ by density arguments.

We comment that as $\mu \rightarrow 0$ the equilibrium state converges to the ground state of the displaced harmonic oscillator, whose Hamiltonian is $H$. However, the rate of convergence to the equilibrium state decreases and in the limit there is no such convergence. This theorem provides a justification for regarding $\mu$ as being a parameter describing the degree of external damping.

Acknowledgements. The author would like to thank A. Jaffe and J. Cannon for many valuable conversations. 


\section{References}

1. Davies,E.B.: Quantum stochastic processes. Commun. math. Phys. 15, 277-304 (1969).

2. - Quantum stochastic processes II. To appear.

3. - Lewis, J.T.: An operational approach to quantum probability. Commun. math. Phys. 17, 293-260 (1970).

4. Klauder,J.R., Sudarshan,E.C.G.: Fundamentals of quantum optics. New York: W. A. Benjamin, Inc. 1968.

5. Lanford, O.E.: Construction of quantum fields interacting by a cut off Yukawa coupling. Princeton University thesis 1966.

E. B. Davies

Massachusetts Institute of Technology

Department of Mathematics

Cambridge, Mass., 02139, USA

Permanent address:

Mathematical Institute

24-29 St. Giles

Oxford, England 